\title{
Nanocharacterization and Control of Supported Ni Nanocatalysts for Partial Oxidation of Methane
}

\author{
R.Banerjee*, S.Chenna* and P.A.Crozier* \\ * School of Materials, Arizona State University, 85287-1504, Tempe, AZ, USA
}

Oxide supported metal catalysts play a critically important role in the field of energy. The effectiveness of the catalyst depends on its ability to create and maintain a large number of active sites for the reaction of interest; it often requires a uniform distribution of small metal particles over a high surface area support. The incipient wetness technique is a popular approach for preparing supported metal catalysts. We are employing a combination of in situ and ex situ electron microscopy to develop a fundamental understanding of this process during the preparation of supported Ni catalysts for partial oxidation of methane [1]. We work on well-defined high surface area and planar supports in order to develop protocols for synthesizing nanocatalysts with precisely controlled morphology. These supports are also well suited for using electron microscopy observations to elucidate the fundamental processes responsible for morphological changes. [2].

In the present work, we employ planar silica and amorphous silica spheres (prepared by Stober's method [3]) and crystalline $\mathrm{MgO}$ cubes [4] as model supports to study the evolution of the $\mathrm{Ni}$ particles during catalyst preparation and partial oxidation of methane. Solutions of nickel nitrate hexahydrate, $\mathrm{Ni}\left(\mathrm{NO}_{3}\right)_{2} \cdot 6 \mathrm{H}_{2} \mathrm{O}(99.999 \%$, Sigma Aldrich, USA) were employed to provide the metal salt for impregnations. Two sets of $\mathrm{Ni} / \mathrm{SiO}_{2}$ samples were prepared using the incipient wetness technique to compare a conventional preparation to that of a modified preparation. In the conventional method, aqueous impregnation was carried out under room conditions followed by calcination at $300^{\circ} \mathrm{C}$ for $3 \mathrm{hrs}$ and reduction at $400^{\circ} \mathrm{C}$ for $3 \mathrm{hrs}$ in $5 \% \mathrm{H}_{2} / \mathrm{Ar}$. In the modified version, impregnation was performed using ethanol in a saturated ethanol atmosphere in a glove box followed by direct reduction at $400^{\circ} \mathrm{C}$ for $3 \mathrm{hrs}$ in $5 \% \mathrm{H}_{2} / \mathrm{Ar}$. In the latter modified case, the samples were constantly mixed in a mortar for 10 mins during impregnation and dried at $120^{\circ} \mathrm{C}$ for 2 hours. Nanoanalysis at each stage of the preparation was performed on a JEOL JEM-2010F scanning transmission electron microscope (STEM) or followed in situ in an environment FEI Tecnai F20.

Fig 1 shows typical STEM images of the resulting precursor prepared in the conventional way. Most spheres have either no precursor present (Fig 1a) or show large agglomeration (Fig 1b) of the precursor resulting in poor metal dispersion in the final catalyst after calcination and reduction (Fig 1c). For samples prepared in the modified way, we observe the presence of thin precursor layers on most of the silica spheres (Fig 2a, 2b) and a uniform and high dispersion of metal after reduction (Fig 2c). Better dispersion in the modified version can be explained as follows. The lower surface tension of ethanol compared to water facilitates wetting of the silica spheres and the saturated ethanol vapor ambient prevents drying of ethanol during mixing. Our work on planar supports demonstrated the importance of ambient vapor pressure for maintaining a wet slurry during the mixing step of the impregnation allowing each silica particle to be uniformly coated with salt. A direct reduction step was used to prevent coarsening of the nanoparticles during calcination [5]. We will present corresponding observations for $\mathrm{Ni}$ supported on $\mathrm{MgO}$. We will also discuss the stability 
of the metal particle dispersion and the morphology of the active catalyst based on measurement from both ex-situ and in-situ after and during partial oxidation of methane.

\section{References:}

[1] B.C. Enger, R. Lodeng, A. Holmen, Applied Catalysis A: General 346 (2008) 1

[2] A.K. Datye, Ultramicroscopy 25 (1998) 203-208

[3] W.Stober, A.Fink, E.Bohn, Journal of colloid and interface science, (1968), 62

[4] G. Glaspell et al., Journal of Physical Chemistry B, 110 (2006) 21387

[5] C. Louis, Z. X. Cheng, M. Che, Journal of Physical Chemistry, 97 (1993), 5703

[6] The support from the National Science Foundation (NSF-CBET-0553445) and the use of TEM at the John M. Cowley Center for High Resolution Microscopy at Arizona State University are gratefully acknowledged.
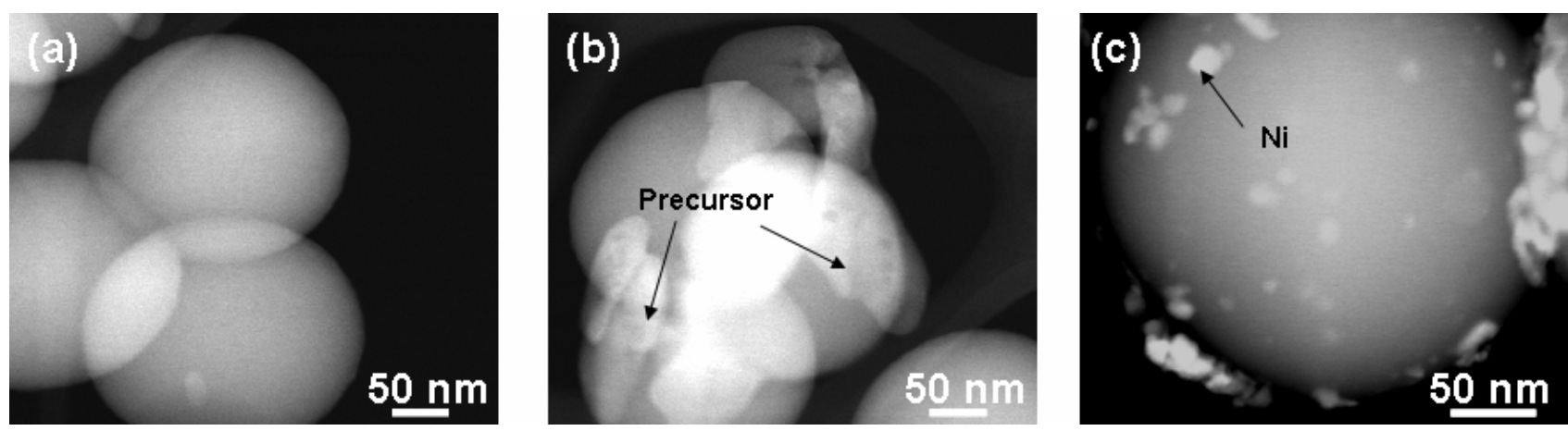

Fig 1: STEM image from conventional preparation demonstrating heterogeneity of nickel nitrate coverage on individual silica spheres showing (a) absence of precursor and (b) excess precursor coverage (c) Metal particles after calcination followed by reduction.
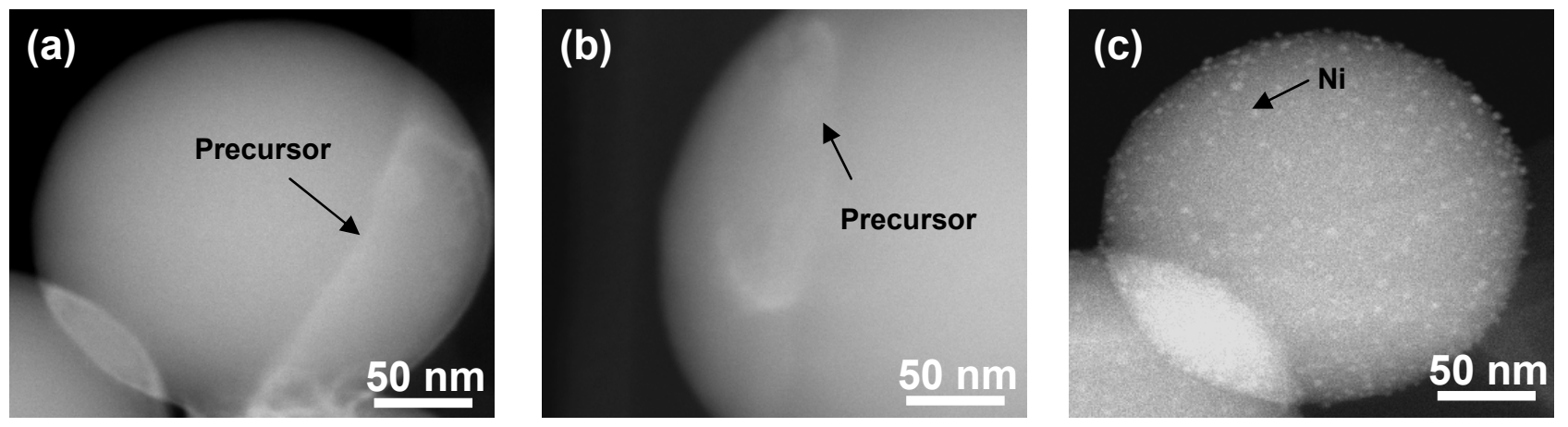

Fig 2: STEM image of nickel nitrate precursor on Silica prepared by modified way (a) and (b) showing presence of thin layer of precursor (c) After reduction 